\title{
Utilizing Google Trends to Assess Worldwide Interest in COVID-19 and Myocarditis
}

\author{
Agam Bansal $^{1}$ - Samiksha Gupta ${ }^{1} \cdot$ Vardhmaan Jain $^{1} \cdot$ Ashish Kumar ${ }^{1} \cdot$ Allan Klein $^{1}$
}

Received: 18 November 2020 / Accepted: 24 November 2020 / Published online: 11 January 2021

(C) Springer Science+Business Media, LLC, part of Springer Nature 2021

COVID-19 was declared a pandemic by World Health Organization on 11th March 2020. Currently, there are 39.8 million cases and 1.11 million deaths reported from the disease worldwide [1]. Myocarditis is an inflammatory disease of the heart characterized by inflammatory infiltrates and myocardial injury without an ischemic etiology [2]. Severe acute respiratory syndrome coronavirus 2 (SARS-CoV-2) related myocarditis is thought to be secondary to "cytokine storm" or direct viral injury [3]. Myocarditis can have a wide spectrum of clinical presentation ranging from mild symptoms including fatigue, chest pain to life threatening complications including cardiogenic shock, ventricular arrhythmias or sudden cardiac death. The prevalence of myocarditis in COVID patients is unclear, however reports suggest around $7 \%$ of deaths in COVID patients is secondary to myocarditis [2]. We aimed to assess the worldwide interest in myocarditis in the COVID era using Google Trends.

Accounting for $75 \%$ of internet searches worldwide, Google Trends is a useful platform to observe trends over time and global and regional variations in interest on a particular topic [4]. Google trends measures "popularity" or "interest" of a particular search query in relative search volume (RSV). RSV can be measured in two ways [5]: first is the popularity of a search term in a given week relative to other weeks in the mentioned time period within a geographic region. The most popular week has a RSV of 100 and all other weeks are reported relative to the most popular week on a scale from 0 to 99 . For example, an RSV of 10 would indicate that search term was $10 \%$ as popular as

This article is part of the Topical Collection on Education \& Training

\section{Allan Klein}

kleina@ccf.org

1 Department of Cardiovascular Medicine and Department of Thoracic and Cardiovascular Surgery Cleveland Clinic, Center for Diagnosis and Treatment of Pericardial Disease, Cleveland Clinic, 9500 Euclid Ave., Desk J1-5, Cleveland, OH, USA it was in the most popular week. Second way is the popularity of a search term over the mentioned time period and can be compared between geographic regions. The region with the highest proportion of searches will have a RSV of 100 and other geographic areas will have RSV in relation to this. We performed Google Trends search on 17th October, 2020 to observe trends in the internet searches for "Myocarditis" from 1st January, 2018 until 17th October 2020. We also conducted a Google Trend analysis in the COVID era from 1st February, 2020 until 17th October, 2020 for the following terms: "covid myocarditis", "covid-19 myocarditis", "coronavirus myocarditis". Geographic trends for the search query "covid myocarditis" were plotted across the world and the United States.

There was a significant rise in the search hits for "myocarditis" from 2018 to October 17th 2020, beginning around August 2020, peak in September 2020 followed by decline in October 2020 (Fig. 1a). Amongst the COVID and myocarditis related terminologies, "covid myocarditis" was the most popular search term from Feb to October 2020 (Fig. 1b). Figure 1c shows the search hits across the globe for "covid myocarditis". The top 5 countries with highest search hits were Ireland followed by United States, Canada, United kingdom and India. In the United States, RSV for "covid myocarditis" was highest in New Hampshire followed by Massachusetts, Oklahoma, Oregon and Kansas (Fig. 1d).

In our study, we used a novel, publicly available tool to assess the interests in the search terms related to COVID and myocarditis. The increase in relative search volume of "myocarditis" and "covid myocarditis" in COVID era around August to September 2020 could be attributed to increased awareness and knowledge about the COVID related cardiovascular complications including myocarditis over time or underreported cases of SARS-CoV-2 induced myocarditis.

The association between COVID and myocarditis may have been noted prior to first case report published in early April [6], as evident by small increases in search in "covid myocarditis" around March to April (Fig. 1b). 
a

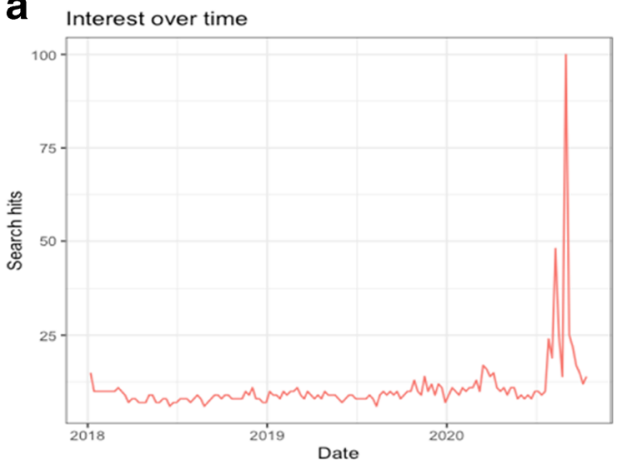

C

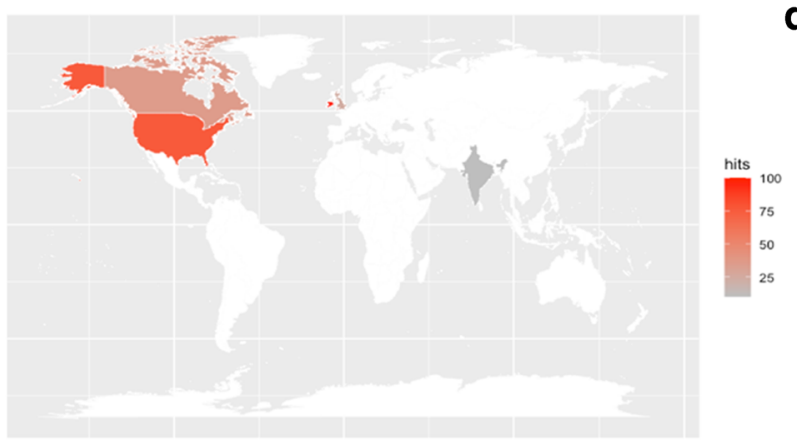

Fig. 1 a Relative search volume of "myocarditis" from 2018 to 17th October, 2020. b Relative search volume of "covid myocarditis", "covid-19 myocarditis" or "coronavirus myocarditis" from February to

There are several limitations to our study: 1) Google trends does not measure the prevalence of the condition but measures the relative interest in search terms which is influenced by factors associated with internet usage; 2) inability to measure the absolute number of searches within a region; 3) governmental control of search engines and language barriers could also impact the full spectrum of searches across the region [5]; and 4) lack of information from other search engines. Further studies are needed to determine whether Google Trends is a reliable tool for investigating trends in public interest in cardiovascular disorders. In conclusion, google trend analysis reflects a continued worldwide interest in the association between COVID and myocarditis.

\section{Compliance with ethical standards}

Conflict of interest The authors declare no conflicts of interest.

Ethical approval This article does not contain any studies with human participants or animals performed by any of the authors.

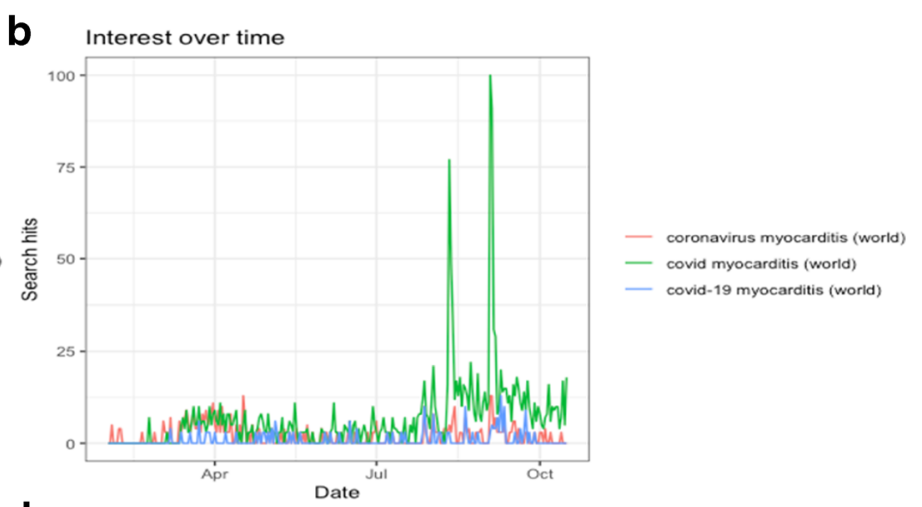

d

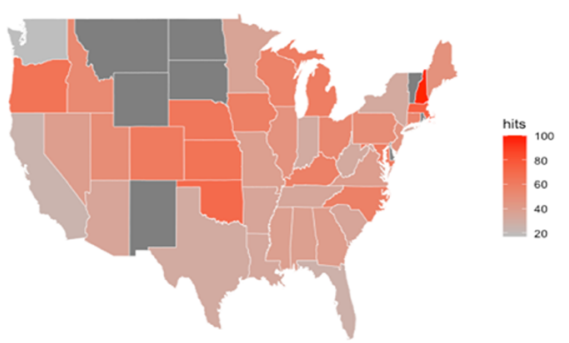

October 17th 2020. c Worldwide interest in the search term "covid myocarditis". d US state wise interest in the search term "covid myocarditis"

\section{References}

1. Coronavirus disease 2019 [Internet]. [cited 2020 October 17]. Available from: https://www.who.int/emergencies/diseases/novelcoronavirus-2019. Accessed 17 Oct 2020

2. Siripanthong, B., Nazarian, S., Muser, D., Deo, R., Santangeli, P., Khanji, MY., et al. Recognizing COVID-19-related myocarditis: The possible pathophysiology and proposed guideline for diagnosis and management. Heart Rhythm. 17:1463-1471, 2020

3. Pirzada, A., Mokhtar, A.T., Moeller, A.D., COVID-19 and Myocarditis: What Do We Know So Far? CJC Open. 2:278-285, 2020.

4. Dey, M., Zhao, S.S., COVID-19 and Kawasaki disease: an analysis using Google Trends. Clin Rheumatol. 39:2483-2484, 2020.

5. Flanagan, R., Kuo, B., Staller, K., Utilizing Google Trends to Assess Worldwide Interest in Irritable Bowel Syndrome and Commonly Associated Treatments. Dig. Dis. Sci. 2, 2020

6. Zeng, J.-H., Liu, Y.-X., Yuan, J., Wang, F.-X., Wu, W.-B., Li, J.-X., et al. First case of COVID-19 complicated with fulminant myocarditis: a case report and insights. Infection. 48:773-777, 2020.

Publisher's Note Springer Nature remains neutral with regard to jurisdictional claims in published maps and institutional affiliations. 